\title{
Avaliação Heurística de Usabilidade em Museus Virtuais de Moda Baseados na Web
}

\section{Heuristic Avaliation of Usability in Web-Based} Fashion Museums

Aline Padaratz ${ }^{1}$ 


\section{Resumo}

Este artigo teve como objeto de pesquisa a avaliação de acervos digitais de museus de Moda baseados na web. Foi apresentada primeiramente uma contextualização dos museus no mundo virtual, identificando os tipos de sites museológicos existentes e os conceitos gerais de usabilidade. Em seguida, através de um estudo comparativo das heurísticas e recomendações de usabilidade de Nielsen, Bastien \& Scapin, e Jordan, foram identificadas as mais adequadas para aplicação neste tipo de acervo, principalmente no que se refere à interação do usuário e ao estímulo de processos cognitivos para estabelecer estes espaços virtuais como locais de aprendizado, sem depender do espaço físico. Optou-se pelas heurísticas de Nielsen, que foram então aplicadas em uma avaliação da interface do site do Australian Dress Register, vinculado ao Powerhouse Museum Science + Design. Os resultados preliminares permitiram identificar as recomendações de usabilidade mais propícias para avaliar a estrutura e organização de sites de acervos digitais de museus de Moda.

Palavras-Chave: usabilidade, heurísticas, web, moda, museu.

\section{Abstract}

The research subject of this article was the evaluation of digital collections of web based fashion museums. The contextualization of museums in the virtual world was first presented, identifying the types of museum sites and general usability concepts. Then, through a comparative study of heuristics and recommendations for usability by Nielsen, Bastien \& Scapin, and Jordan, we identified the most appropriate application for this type of collection, especially with regard to user interaction and cognitive stimulation processes to establish these virtual spaces as places of learning, without relying on physical space. Nielsen's heuristics were chosen, which were then applied in a review of the Australian Dress Register site interface, linked to the Powerhouse Museum Science + Design. These preliminary results showed the most favorable usability recommendations to assess the structure and organization of digital collections of fashion museum websites.

Keywords: usability, heuristics, web, fashion, museum.

ISSN: $1808-3129$

1 Graduada em Moda pela Universidade do Estado de Santa Catarina, Brasil, aline.padaratz@gmail.com 


\section{INTRODUÇÃO}

A missão principal de qualquer museu é disseminar informação, comunicar, e educar o público leigo, e a disponibilização do acervo digital na web pode auxiliar muito este processo. A presença online do museu não apenas amplia o seu público, ao eliminar as barreiras geográficas, mas também tem o potencial de oferecer ao usuário a exploração detalhada dos artefatos que lhe interessam e o aprofundamento de seus contextos (STEWART, 2009, p.14-5). Assim, o museu virtual aumenta o alcance de determinado acervo não apenas ao público geral, mas também a um público especializado - no exemplo de um museu de Moda, a estudantes, pesquisadores e profissionais do ramo - ao proporcionar uma experiência enriquecedora de pesquisa, sem prejuízo à conservação dos artefatos.

No entanto, os sites de museus de Moda e artefatos têxteis em geral preocupam-se apenas com a comunicação e criação de uma identidade visual para a instituição, com objetivo de atrair visitantes ao museu físico em vez de proporcionar um mundo virtual à parte aos usuários para a produção de novos conhecimentos (SAIKI e ROBBINS, 2008, p. 831).

O estudo da usabilidade aplicada ao tipo de acervo digital em questão ainda é incipiente, principalmente no que se refere à interação do usuário e ao estímulo de processos cognitivos para estabelecer estes espaços virtuais como locais de aprendizado, sem depender do espaço físico. Assim, percebe-se a importância de identificar diretrizes de usabilidade para museus virtuais de Moda e para tal, torna-se necessário identificar a maneira mais adequada de disponibilizar o acervo de um museu virtual de Moda que proporcione uma experiência satisfatória ao usuário.

Como etapa preliminar à elaboração destas diretrizes será realizado no presente artigo uma exploração do método de avaliação heurística em um website de acervo de Moda, o Australian Dress Register, vinculado ao Powerhouse Science + Design Museum . O artigo está estruturado da seguinte forma: primeiramente, são discutidas as principais recomendações dentro da literatura especializada e identificadas as diferenças e equivalências entre três (03) conjuntos de heurísticas. Em seguida, um destes conjuntos é utilizado para a avaliação do site mencionado como estudo de caso, concluindo com a identificação das heurísticas mais adequadas para a avaliação deste tipo de website.

\section{USABILIDADE EM MUSEUS VIRTUAIS DE MODA BASEADOS NA WEB}

\subsection{Categorias de sites museológicos}

Existem basicamente três tipos de sites museológicos: folhetos eletrônicos, museus no mundo virtual e museus realmente virtuais (PIACENTE apud HENRIQUES, 2004, p.05-7). O primeiro é o tipo mais comum encontrado na internet, e costuma ter como objetivo divulgar informações básicas sobre a instituição, como localização, horário de funcionamento, histórico do museu, entre outras. 
Os museus no mundo virtual constituem sites que dispõem informações mais detalhadas sobre o acervo, muitas vezes disponibilizando bases de dados de seus objetos: "O site acaba por projetar o museu físico na virtualidade e muitas vezes apresenta exposições temporárias que já não se encontram mais montadas em seu espaço físico, fazendo da Internet uma espécie de reserva técnica de exposições" (HENRIQUES, 2004, p.05).

A terceira categoria de site é caracterizada pela interatividade, ou seja, pelo envolvimento do público. Muitas vezes, o museu virtual funciona como complemento do museu físico - quando este existe - e não apenas reproduz o seu conteúdo, mas também proporciona novas facetas para o mesmo (HENRIQUES, 2004, p.07). Assim, o museu virtual pode apresentar duas configurações: como dimensão virtual de um museu físico (ou seja, existente também no mundo real) ou como museu puramente virtual (HENRIQUES, 2004, p.11-12). Por causa disso, segundo Henriques (2004), esta categoria se diferencia das outras por deixar de constituir meramente um site de museu e sim, um museu virtual.

A discussão teórica sobre o conceito de museu virtual ainda é bastante incipiente e a maioria dos sites que se dizem museus virtuais não exploram as potencialidades oferecidas por este meio. Considera-se para os fins deste artigo que o site selecionado para análise se enquadra dentro da categoria de museu virtual por não apresentar local físico (apesar de vinculado a um museu real), mas sim, um espaço na web que agrega acervos de diversos museus de moda conveniados, assim como acervos de coleções particulares, em um único espaço virtual.

\subsection{Heurísticas de Usabilidade}

Em termos gerais, usabilidade está associada à facilidade de uso de um produto, ou seja, a habilidade de um indivíduo em usar algo e realizar uma tarefa com sucesso (TULLIS e ALBERT, 2008, p.04). Os princípios de usabilidade estruturam a avaliação de uma interface, e neste contexto prático, são denominados de heurística. Segundo Preece et al (2007, p.48), o termo heurística "[...] enfatiza que algo deve ser feito com esses princípios, quando aplicados a um dado problema. Em particular, precisam ser interpretados no contexto do design, utilizando-se de experiências já realizadas [...]". Ou seja, para cada objeto a ser avaliado é necessário moldar os conjuntos de heurísticas e recomendações para suas especificidades.

Para este trabalho, foram selecionados três conjuntos de heurísticas, para um estudo comparativo a fim de identificar qual deles se encontra mais adequado para aplicação em museus virtuais de Moda baseados na web. São eles as Heurísticas de Usabilidade de Nielsen (1993), os Critérios Ergonômicos de Bastien \& Scapin (1997), e as Recomendações de Usabilidade de Jordan (2001), que podem ser verificados no Quadro 1. 
Quadro 1 - Heurísticas de Nielsen, Bastien \& Scapin, e Jordan

\begin{tabular}{|l|l|l|}
\hline $\begin{array}{l}\text { Heurísticas de Usabilidade de } \\
\text { Nielsen (1993) }\end{array}$ & $\begin{array}{l}\text { Critérios Ergononmicos de Bas- } \\
\text { tien \& Scapin (1997) }\end{array}$ & $\begin{array}{l}\text { Rencomendações de usabilicade } \\
\text { de Jordan (2001) }\end{array}$ \\
\hline 1) Diálogo simples e natural & 1) Condução & 1) Coerencia \\
\hline 2) Compatibilidade & 2) Carga de Trabalho & 2) Compatibilidade \\
\hline $\begin{array}{l}\text { 3) Reconhecimento em vez de } \\
\text { memorização }\end{array}$ & 3) Controle expl;icito & $\begin{array}{l}\text { 3) Consideração sobre a habilidade } \\
\text { do usuário }\end{array}$ \\
\hline 4) Consistencia e padrões & 4) Adaptabilidade & 4) Retorno das ações/feedback \\
\hline 5) Feedback & 5) Gestão de erros & 5) Prevenção de erro \\
\hline 6) Controle do usuário & 6) Coerencia & 6) Controle do usuário \\
\hline 7) Atalhos & 7) Significados dos códigos e de- & 7) Clareza visual \\
\hline 8) Boas mensagens de erros & 8) Compatibilidade & $\begin{array}{l}\text { 8) Priorização da funcionalidade da } \\
\text { infomaçãao }\end{array}$ \\
\hline 9) Prevenção de erros & $\begin{array}{l}\text { 9) Transferencia adequada de } \\
\text { tecnologia }\end{array}$ \\
\hline 10) Ajuda e documentação & & 10) Explicitação \\
\hline
\end{tabular}

Fonte: A autora

Os oito critérios ergonômicos de Bastien \& Scapin foram estabelecidos para o âmbito da $\mathrm{HCl}$ (Interação humano-computador), com fins de identificar e classificar qualidades e problemas em softwares interativos (KAZEDANI, p.01).

Em termos de Condução, os aspectos identificados por Bastien \& Scapin abrangem questões relativas à orientação do usuário de forma a instruir e guiar sua interação com a interface, como o agrupamento e distinção de itens por localização e formato, feedback imediato e legibilidade. Uma boa condução facilita o aprendizado e uso de um sistema, melhorando a performance e reduzindo erros (BASTIEN \& SCAPIN, 1997, p.222). A redução de erros está relacionada, também, à redução da Carga de Trabalho do usuário. Este segundo critério inclui os aspectos cognitivos que interferem na eficiência do usuário para cumprir determinada tarefa, entre eles, a brevidade das ações necessárias para cumprir uma tarefa e a densidade das informações apresentadas ao usuário. O controle que o usuário possui sobre suas ações e sobre o processamento do sistema também é outro fator que limita a ocorrência de erros e reduz ambiguidades do sistema, permitindo maior aceitação por parte do usuário (BASTIEN \& SCAPIN, 1997, p.225).

A Adaptabilidade do Sistema se refere à flexibilidade de opções oferecidas ao usuário para a realização de uma mesma tarefa de acordo com suas preferências e da consideração de sua experiência com a interface. A adaptação do comportamento da interface às necessidades do usuário, diversificando as possibilidades de ações para o mesmo objetivo, permite que o usuário encontre o procedimento ideal para suas particularidades e evita efeitos negativos na interação. Por se tratar de critérios estabelecidos inicialmente para $\mathrm{HCl}$, o critério de gestão de erros estabelecido por Bastien \& Scapin se refere às maneiras de prevenção e recuperação de erros deste contexto, como a qualidade das mensagens de erro, por exemplo.

Coerência diz respeito a padronização de elementos de design da interface quando aplicados em contextos diferentes, condição que torna o sistema mais pre- 
visível e facilita o aprendizado do uso. A falta de consistência de aspectos como formatos de menu e layout, por exemplo, é um dos principais motivos de rejeição do usuário. O critério de Significação dos Códigos trata da adequação entre um termo e sua referencia. Esta relação, quando significativa, facilita a recordação e o reconhecimento por parte do usuário, sendo necessário que lhe sejam familiares e não arbitrários. Por fim, o critério de Compatibilidade se refere a concordância entre as características psicológicas do usuário (como hábitos e idade, por exemplo) e da tarefa, assim como a organização das entradas, saídas e diálogos do sistema.

Jordan (2001) recomenda dez princípios para um design "usável": Coerência, Compatibilidade, Considerações sobre as Habilidade do Usuário, Retorno das Ações (Feedback), Prevenção de Erro e Recuperação, Controle do Usuário, Clareza Visual, Priorização da Funcionalidade e da Informação, Transferência Adequada de Tecnologia, e Explicitação. Como é possível notar, algumas destas recomendações se assemelham às de Bastien \& Scapin. No entanto, ao contrário destes, as de Jordan não foram formuladas dentro de um contexto específico, sendo mais fácil aplicá-las a uma diversidade maior de interfaces e produtos.

Alguns destes critérios apresentam apenas algumas variações às de Bastien \& Scapin, como o de Compatibilidade que, para Jordan (2001) trata de adequar o método de operação do produto às expectativas do usuário baseadas em seu conhecimento de outros produtos e do "mundo externo" (JORDAN, 2001, p.27), e não apenas às suas características pessoais.

A recomendação de Feedback de Jordan é apresentada dentro do critério de Condução de Bastien \& Scapin. Para ambos, o Feedback se refere ao retorno das ações do usuário, ou seja, o reconhecimento por parte do sistema sobre as ações do usuário e a indicação significativa sobre os resultados destas ações (JORDAN, 2001, p.29). Do mesmo modo, as recomendações de Jordan de Prevenção de Erro e Recuperação, assim como a de Controle do Usuário, se inserem dentro dos critérios de Gestão de Erros e Controle Explícito de Bastien \& Scapin, respectivamente. A recomendação Explicitação de Jordan é semelhante em alguns aspectos à de Significado dos Códigos e Denominações de Bastien \& Scapin, onde as funções de um sistema que são representados por ícones devem apresentar indícios explícitos sobre estas funções. Em outros contextos, o critério de Explicitação de Jordan se refere a projetar um produto de maneira a deixar claro para o usuário como este deve ser operado.

As recomendações de Jordan que mais diferem das de Bastien \& Scapin são as de Consideração sobre a Habilidade do Usuário, Clareza Visual, Priorização da Funcionalidade e da Informação, e Transferência Adequada de Tecnologia. A primeira leva em conta a demanda das habilidades ou recursos que o usuário dispõe na interação com o produto, como o uso dos sentidos de visão, audição e tato. Segundo Jordan, é importante que durante o uso de um produto não haja sobrecarga de uma das habilidades do usuário em relação a outras para que não ocorram problemas de usabilidade (JORDAN, 2001, p.27).

As recomendações de Clareza Visual e Priorização da Funcionalidade e da Informação buscam modos de evitar confusão na disposição dos elementos em uma determinada interface. A primeira ocupa-se principalmente com a aplicação de elementos gráficos, como o uso de cores, o tamanho e tipo de fontes, e o posiciona- 
mento dos elementos, de maneira a tornar a leitura mais fácil e rápida. A segundo trata de tornar mais fácil o acesso às funções e informações que sejam mais importantes para o usuário. Assim, funções de uso mais frequente devem estar dispostas na interface através de estruturas hierárquicas adequadas (JORDAN, 2001, p.34-5). No caso da interface de um website, por exemplo, isto pode ser realizado através de menus e sub-menus significativos.

A Transferência Adequada de Tecnologia é a última das recomendações de Jordan que diferem das de Bastien \& Scapin, e aborda a aplicação de tecnologias desenvolvidas para fins específicos em outros contextos, diferentes do original.

Assim como os outros autores abordados anteriormente, Nielsen (1993) também insere o Feedback como um de seus principais critérios. Assim, todos os autores consideram a importância de manter o usuário informado sobre o funcionamento do sistema, sendo que Nielsen salienta também que isto deve ocorrer dentro de um limite de tempo razoável. Outros critérios que Nielsen apresenta em comum com os outros autores são o de Controle do Usuário e Liberdade, Consistência e Padrões (que para Jordan e Bastien \& Scapin constitui o critério de Coerência), Flexibilidade e Eficiência de Uso, ou Atalhos (Adaptabilidade, para Bastien \& Scapin e Considerações sobre as Habilidade do Usuário, para Jordan), Boas Mensagens de Erros e Prevenção de erros. Nielsen compartilha também com Jordan o critério de Compatibilidade do Sistema com o Mundo Real, ou Falar a Linguagem do Usuário - a adoção pelo sistema de conceitos familiares ao usuário, seguindo convenções do mundo real e fazendo com que as informações apareçam em uma ordem natural e lógica (NIELSEN, 1993). Este critério é semelhante em alguns aspectos ao de Significado dos Códigos e Denominações de Bastien \& Scapin.

O critério que Nielsen apresenta que os outros autores não consideram especificamente é o de Ajuda e Documentação. Apesar de Nielsen frisar que o ideal seja que a interface não necessite de instruções para seu uso, em alguns casos é necessário fornecer ajuda ao usuário - e esta deve estar disponível de maneira sucinta, direcionada à tarefa específica, de fácil acesso e listada passo-a-passo (NIELSEN, 1993).

A partir da discussão apresentada nesta sessão, cujos resultados podem ser visualizados no Quadro 2, concluiu-se que as heurísticas de Nielsen são as mais completas, neste momento, para uma análise inicial da interface de sites de museus de Moda. Desta forma, o próximo item dispõe a avaliação do site de um museu com base nas heurísticas de Nielsen. 
Quadro 2 - Equivalências entre as heurísticas de Nielsen, Bastien \& Scapin, e Jordan

\begin{tabular}{|l|l|l|}
\hline $\begin{array}{l}\text { Heuristicas da Usabilidade de Nielsen } \\
(1993)\end{array}$ & $\begin{array}{l}\text { Critérios Ergonomicos de Bastien \& } \\
\text { Scapin (1997) }\end{array}$ & $\begin{array}{l}\text { Recomendações da Usabilidade de } \\
\text { Jordan (2001) }\end{array}$ \\
\hline Diálogo simples e natural & Condução & Clareza visual \\
\hline Falar a linguagem do usuário & Compatibilidade & Compatibilidade \\
\hline Redução de carga mental & Carga de trabalho & $\begin{array}{l}\text { Priorização da funcionalidade e da } \\
\text { informação }\end{array}$ \\
\hline Consistencia e padrões & Coerencia & Coerencia \\
\hline Feedback & Condução & Retorno das ações/feedback \\
\hline Controle do usuário & Controle explicito & Controle do usuário \\
\hline Atalhos & Adaptabilidade & $\begin{array}{l}\text { Consideração spbre a habilidade do } \\
\text { usuário }\end{array}$ \\
\hline Boas mensagens de erros & Gestão de erros & Prevenção de erro e recuperação \\
\hline Prevenção de erros & Gestão de erros & Prevenção de erro e recuperação \\
\hline Compatibilidade & $\begin{array}{l}\text { Significados dos códigos e denomi- } \\
\text { nações }\end{array}$ & Explicitação \\
\hline & & Transferencia adequada de tecnologia \\
\hline Ajuda e documentação & & \\
\hline
\end{tabular}

Fonte: A autora

\subsection{Análise de um Museu Virtual de Moda com base nas heurísti- cas de Nielsen}

\subsubsection{Método utilizado}

A avaliação heurística é uma técnica simples e rápida utilizada no campo dos Fatores Humanos, que envolve a obtenção de opiniões subjetivas a respeito de um objeto ou conceito de design. Ao interagir com o artefato em questão, cabe ao avaliador ou equipe de avaliadores observarem aspectos de usabilidade, qualidade e potencial de erro do projeto (SALMON, 2004, p.470). A avaliação é conduzida com o especialista se colocando como usuário típico do produto, anotando os problemas que encontra utilizando as heurísticas específicas para a interface em questão (PREECE et al, 2007, p.431). É comum que a avaliação seja realizada por uma equipe de cinco especialistas, devido a evidências que sugerem que este número de avaliadores é capaz de identificar até $75 \%$ dos problemas de usabilidade do produto (PREECE et al, 2007, p.431). Para os fins deste artigo, porém, a avaliação foi realizada de maneira individual, a fim de observar alguns aspectos da interface de um acervo digital de Moda através das heurísticas de Nielsen.

\subsubsection{Estudo de caso: site Australian Dress Register}

A interface do site Australian Dress Register, apresenta em sua página inicial (Figura 1) um layout simples e clareza visual. As cores utilizadas são neutras e claras, sendo o laranja aplicado em elementos de destaque, como alguns links e botões do menu principal, ao se passar o mouse por cima. 


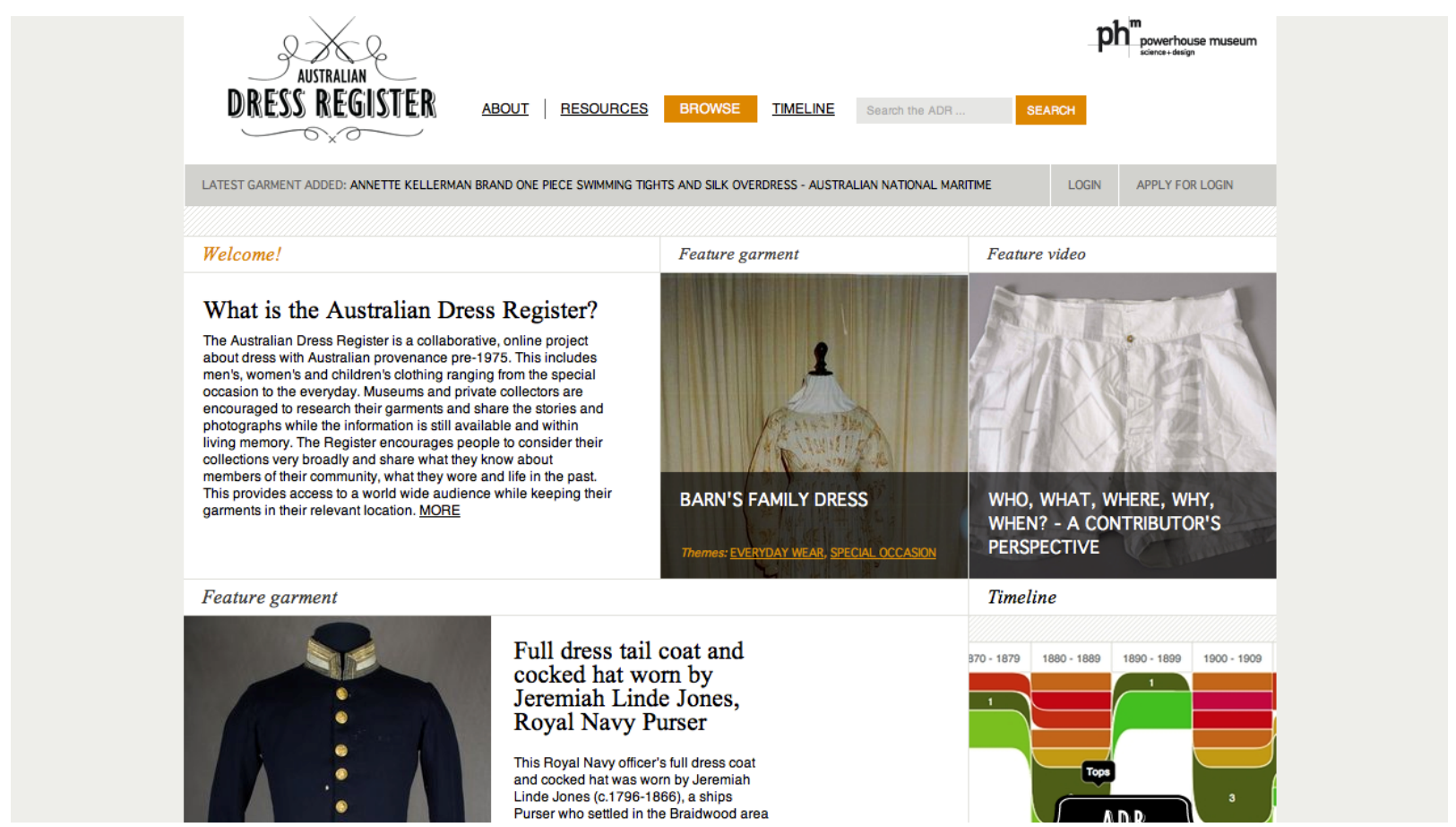

Figura 1 - Interface da página inicial. Australian Dress Register. Disponível em: <http://www.australiandressregister.org/> Acesso em: 22 nov. 2013.

O menu superior é bastante conciso e agrupa as três maneiras disponíveis de pesquisar o acervo: a busca por palavra-chave, por navegação e por uma linha do tempo. Os outros dois itens do menu são About (informações sobre o site) e $R e-$ sources (recursos educacionais). Com exceção da pesquisa por palavra-chave, representada por uma caixa de texto - procedimento convencional em sites de busca - os outros itens do menu são representados por palavras únicas e não apresentam sub-menus em cascata. Assim, priorizam as informações principais e os objetivos do site, deixando claro ao usuário as opções disponíveis. O sistema se utiliza de uma linguagem de fácil compreensão e segue convenções do "mundo real", apresentando a informação de forma lógica e natural (NIELSEN, 1995).

Abaixo do menu há uma barra cinza onde é informada a última peça adicionada ao acervo. Além disso, à direita, há um botão para login e ao lado deste, um botão para solicitação de login. Este três itens constituem links, porém não seguem o mesmo padrão do menu acima - não apresentam sublinhado, mas sim formato de botão. Apesar de todos estes elementos mudarem de cor ao se passar o cursor por cima (o mesmo ocorre com os itens do menu superior), não fica claro, inicialmente, que são "clicáveis", principalmente o item de acervo mais recente. O mesmo padrão de uso do laranja para indicação de links também é aplicado em imagens do resultado de busca (Figura 2). 




Figura 2 - Interface da página de resultados de busca. Australian Dress Register. Disponível em: <http://www.australiandressregister.orgl> . Acesso em: 22 nov. 2014.

Neste caso, ao se passar o mouse por cima da imagem ela é substituída por um retângulo laranja preenchido com texto em branco. Ainda na visualização de resultados de busca, a coluna da esquerda apresenta uma lista de filtros disponíveis - entre eles, instituição, materiais, temas, etc. Ao lado de cada categoria, um sinal de "+" indica a existência de sub-categorias, e ao passar o mouse por cima, também ficam laranja. Assim, em geral, há padronização do laranja para preenchimentos de espaços como indicativo de algo "clicável". No entanto, na página inicial, apesar deste mesmo recurso ser utilizado em imagens em destaque, a cor utilizada é preto em vez de laranja. Esta exceção indica uma falta de consistência no uso das cores na interface do site, segundo a heurística de Nielsen (1993).

Além do menu principal, há na parte inferior do site outros menus que repetem três itens do menu principal: o About, Resources, e Browse. Esta repetição mostra-se desnecessária, desviando do princípio de design minimalista sugerido na primeira heurística de Nielsen (1993). Neste mesmo menu inferior (Figura 3), são incluídos sub-itens que no superior são omitidos. Assim, no item Browse, são apresentados alguns dos filtros já mencionados, além de incluir a opção de pesquisa Timeline como sub-item também. 


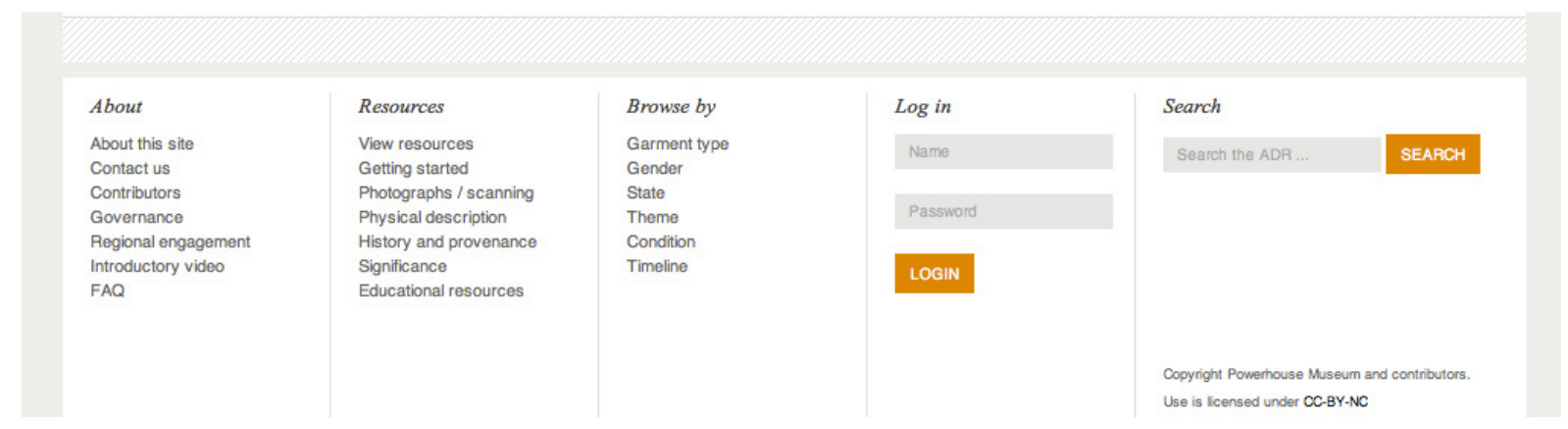

Figura 3 - Menu inferior. Australian Dress Register. Disponivel em: <http://www.australiandressregister.org/> . Acesso em: 22 nov. 2014.

Além disso, são repetidos nesta parte inferior do site, as opções de login (agora com campos para preenchimento, ao contrário de apenas um botão, como na parte superior) e campo de pesquisa por palavra-chave idêntico ao do menu superior. Apesar da repetição poder ocasionar confusão, principalmente pela inclusão da Timeline como filtro da opção Browse e pela disponibilização de apenas alguns filtros neste espaço do site, pode-se dizer que este menu inferior proporciona atalhos a informações importantes porém menos acessadas, além das informações principais. Já no menu superior, percebe-se uma intenção de priorizar o acesso rápido às funções essenciais do site, sem poluição de muitos elementos.

Segundo Nielsen e Loranger (2007), o uso de links diretos na página principal para poucas tarefas de alta prioridade é um dos recursos mais bem-sucedidos utilizados pelo design: "Independentemente da estrutura da arquitetura da informação ou da transparência com que você a representa no seu sistema navegacional, os usuários talvez fiquem perdidos ou impacientes se precisarem navegar por vários níveis. Links diretos encurtam e simplificam isso" (NIELSEN e LORANGER, 2007, p.210). Desta forma há uma hierarquização na disposição do conteúdo do site, além de eficiência de uso.

A apresentação dos elementos da página de um objeto do acervo (Figura 4) mantém a padronização da estrutura geral do site, garantindo a consistência interna do sistema e adequando-se à heurística de mesmo nome proposta por Nielsen (1993). 


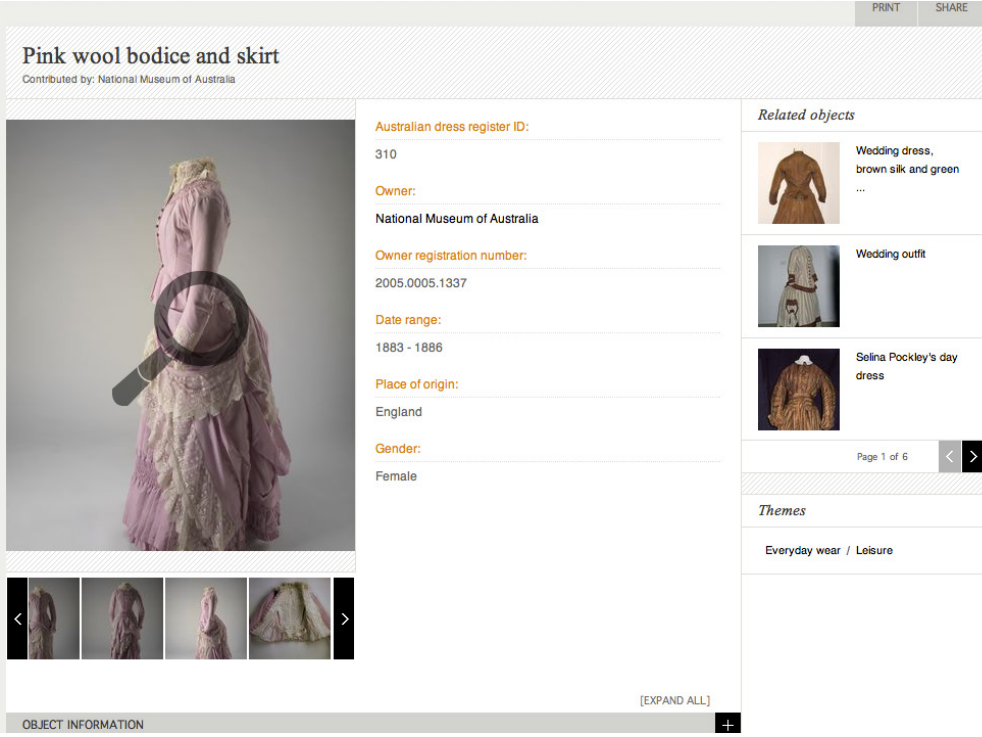

Figura 4 - Interface da página de objeto do acervo. Australian Dress Register. Disponivel em: <http://www.australiandressregister. org/>. Acesso em: 22 nov. 2014.

No entanto, havendo mais informações a serem expostas, alguns recursos foram empregados para limitar o espaço utilizado. Como é possível verificar na Figura 5, as informações sobre o artefato (localizadas logo abaixo da galeria de imagens na interface) foram compactadas e divididas em sub-títulos - entre eles, História e Origem, Detalhes e Aviamentos, Corte, Estado de Conservação, etc.

\begin{tabular}{|c|c|}
\hline OBJECT INFORMATION & \\
\hline HISTORY AND PROVENANCE & \\
\hline TRIMMINGS / DECORATION & \\
\hline Lace & \\
\hline $\begin{array}{l}\text { Cream lace at neckline, outer and inner sleeve cuff. Skirt trimmed with lace } \\
\text { panels to form apron drapery. }\end{array}$ & \\
\hline Tucking & \\
\hline $\begin{array}{l}\text { Fine pleating centre front of skirt, hemline, front and back of the bodice. Gathering } \\
\text { on skirt, neck/shoulder. }\end{array}$ & \\
\hline FIBRE / WEAVE & \\
\hline MANUFACTURE & \\
\hline $\begin{array}{l}\text { All seams are machine sewn. The trimmings, gathering and draping/positioning } \\
\text { of the fabric in the skirt are attached using running stitch and hand sewing. The } \\
\text { boning pieces in the bodice are fully covered and attached by hand using a whip } \\
\text { stitch and hemming stitch. The waist tapes are also attached by hand. The two } \\
\text { finely pleated frill decorative elements at the base of the skirt have been attached } \\
\text { by hand, using running stitch after the pleating had occurred. }\end{array}$ & $\begin{array}{l}\square \text { Hand sewn } \\
\square \text { Machine sewn } \\
\square \text { Knitted } \\
\square \text { Other }\end{array}$ \\
\hline Label & \\
\hline $\begin{array}{l}\text { Attached to the waist tape of the bodice is a gold-stamped label reading DAVID } \\
\text { JONES \& cOMPY/Sydney. On each side of the text is an emblem; one appears to } \\
\text { be a British coat of arms (the text is not legible) and the second reads 'SYDNEY \& } \\
\text { LONDON: }\end{array}$ & \\
\hline CUT & \\
\hline FASTENINGS & \\
\hline STIFFENING / UNING / PADDING & \\
\hline MEASUREMENTS & \\
\hline
\end{tabular}

Figura 5 - Informações sobre objeto do acervo. Australian Dress Register. Disponível em: <http://www.australiandressregister. org/> . Acesso em: 22 nov. 2014. 
Estes itens são listados em barras de cor cinza, onde na extremidade direita foi inserido o sinal de "+", que muda de preto para laranja ao se passar o mouse por cima, seguindo o modelo dos filtros de pesquisa já mencionados. Assim, ao clicar no sinal de "+" o item é expandido e as informações são apresentadas em forma de texto, em geral também dividido em sub-tópicos para evitar grandes blocos de leitura. Esta flexibilidade permite que o usuário detenha o controle sobre quais informações deseja ler a partir das opções da lista, visualizando apenas o que lhe interessa. Assim, percebe-se uma aderência à heurística de controle do usuário, proposta por Nielsen (1993) e os outros autores discutidos. Ainda neste aspecto, o usuário possui liberdade sobre a quantidade de texto que é disposto na página, evitando sobrecarga cognitiva e o uso excessivo da barra de rolagem. Apesar do recurso ser bastante eficiente, ele não fica evidente de imediato, pois por default a página carrega com toda a lista de tópicos expandida, havendo apenas um pequeno link entre colchetes indicando a possibilidade de contrair a lista - só assim percebe-se que o conteúdo é dividido em sessões flexíveis.

Outras questões importantes na página de um objeto do acervo é a indicação clara com relação às opções de visualização das imagens do objeto. Para cada artefato, há uma pequena galeria (Figura 4) que inclui fotografias de diversos ângulos e facetas do objeto, além de outras imagens e documentos complementares que contextualizam a peça. Uma imagem maior é sempre apresentada, com as restantes sendo disponibilizadas em thumbnails (ou seja, em tamanho reduzido), logo abaixo, com setas indicativas para esquerda/direita, demonstrando outros itens a serem visualizados. Ao passar o mouse por cima da imagem maior, surge um ícone representando uma lupa (Figura 4), uma compatibilidade com o "mundo real", onde o objeto lupa é utilizado para fins de ampliação. De fato, ao clicar na lupa uma pequena janela se abre sobre o fundo do site, que fica levemente escurecido (Figura 6). Nesta janela há uma série de comandos de zoom e outras opções de controle, além da indicação clara de como fechar a janela através do " $x$ " - outra convenção já estabelecida na maioria das interfaces humano-computador.

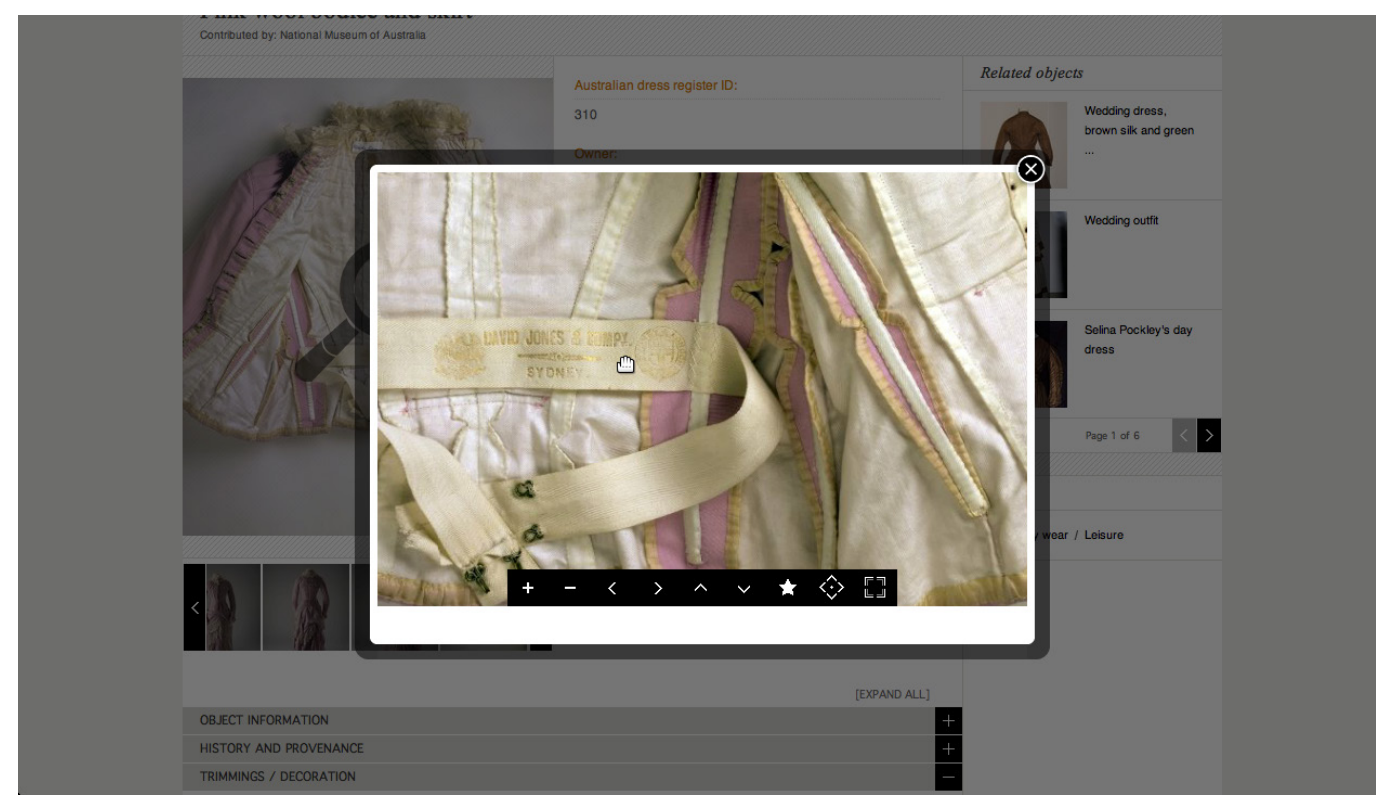

Figura 6 - Janela de visualização de objeto do acervo. Australian Dress Register. Disponível em: < $\underline{\text { http://www.australiandressre- }}$ gister.org/> . Acesso em: 22 nov. 2014. 
Apesar de Nielsen, assim como outros autores, frisar que o ideal em uma interface é que esta seja intuitiva e não necessite de explicação sobre seu funcionamento, o Australian Dress Register disponibiliza na sessão About do site, um vídeo introdutório sobre o site. O vídeo explica os recursos disponíveis, as maneiras de realizar buscas no acervo e formas de participar do projeto. Assim, a presença do vídeo explicativo ajuda o usuário não familiarizado com o conceito novo proposto pelo site, que se aproxima do museu virtual já descrito anteriormente.

\section{CONCLUSÕES}

Este artigo constituiu um exercício inicial de avaliação de usabilidade para interfaces de acervos digitais de museus de Moda baseados na web, com o propósito de identificar alguns aspectos fundamentais na avaliação deste tipo de site. Percebeu-se, através da análise comparativa de heurísticas de usabilidade de Bastien \& Scapin, Jordan, e Nielsen, que muitas delas se aproximam conceitualmente, havendo convergências e divergências sutis dependendo do contexto específico de cada autor. Foi possível notar que, apesar das recomendações de Jordan serem mais gerais e aplicáveis a uma variedade maior de interfaces, a lista de Nielsen demonstrou maior proximidade com o caso específico de interfaces da web, sendo por isso escolhida para ser aplicada na análise.

Assim, através da avaliação do site Australian Dress Register, percebeu-se que entre as dez heurísticas de Nielsen, sete se apresentaram como mais adequadas para a avaliação da interface de acervos digitais de museus de Moda: feedback, compatibilidade, controle do usuário, consistência, atalhos, diálogo simples e natural, ajuda e documentação.

Os demais critérios, inclusive os presentes nas listas dos outros autores abordados também se mostram interessantes para aplicação em acervos digitais de museus de Moda e, apesar de não terem sido utilizadas para a avaliação do site Australian Dress Register, poderão ser úteis para futuros estudos mais aprofundados. 


\section{REFERÊNCIAS BIBLIOGRÁFICAS}

ANDRADE, Juliana Filipa Dias. O museu na era da comunicação online. 2008. 64 f. Dissertação (mestrado) - Universidade do Minho. Disponível na internet por http em: <http://repositorium.sdum.uminho.pt/handle/1822/9524>. Acesso em 31 jan. 2014.

AUSTRALIAN DRESS REGISTER.

Disponível na internet por http em: <http://www.australiandressregister.org>. Acesso em 10 nov. 2013.

BEIRÃO FILHO, José Alfredo. Criação e compartilhamento do conhecimento da área de moda em um sistema virtual integrado de informações. 2011. 166 f. Tese (doutorado). Universidade Federal de Santa Catarina, Programa de Pós-graduação em Engenharia e Gestão do Conhecimento.

BASTIEN, Christian; SCAPIN, Dominique. Ergonomic criteria for evaluating the ergonomic quality of interactive systems. Behaviour $\boldsymbol{\&}$ Information Technology. Londres: v.16, n.4/5, p.220-231, 1997.

HENRIQUES, Rosali. Memória, museologia e virtualidade: um estudo sobre o Museu da Pessoa. 2004. 42 f. Dissertação (mestrado). Universidade Lusófona de Humanidades e Tecnologias. Disponível na internet por http em: <http://www.museologia-portugal.net/files/upload/mestrados/rosali_henriques_1.pdf>. Acesso em 15 nov. 2014.

JORDAN, Patrick W. An Introduction to Usability. Londres: Taylor \& Francis, 2001.

KAZEDANI, Milene. Recomendações de Usabilidade. Disponível na internet por http em: <http://corais.org/node/491> . Acesso em 7 nov. 2013.

KIRKLAND, A.; LESK, M.; STEFFMAN, A. Context for costumes: faceted access to historic costumes. Proceedings of the 2010 International Conference on Electronic Visualisation and the Arts, p.59-65, 2010. Disponível na internet por http em:

< http://dl.acm.org/citation.cfm?id=2227191>. Acesso em: 22 set. 2013.

MARTIN, K.; LIN, X; LUNIN, L. User centric design and implementation of a digital historic costume collection. Proceedings of the American Society for Information Science and Technology, v.40, n. 1, p.280-90, 2005. Disponível em: <http://doi. wiley.com/10.1002/meet.1450400135> . Acesso em: 19 set. 2013.

NIELSEN, Jakob. Usability Engineering. San Diego: Academic Press, 1993. 
NIELSEN, Jakob. 10 Usability Heuristics for User Interface Design, 1995. Disponível em: < http://www.nngroup.com/articles/ten-usability-heuristics/> . Acesso em: 10 nov. 2014.

NIELSEN, Jakob; LORANGER, Hoa. Usabilidade na Web: Projetando Websites com qualidade. Rio de Janeiro: Elsevier, 2007.

PREECE, Jennifer; ROGERS, Yvone; SHARP, Helen. Design de Interação: Além da interação homem-computador. Porto Alegre: Bookman, 2007.

SAIKI, Diana; ROBBINS, Audrey. Trends in information categories on costume and textile collection web sites. The Electronic Library, v.26, n.6, 2008. Disponível em:

<http://www.emeraldinsight.com/journals.htm?articleid=1753954\&show=abstract>. Acesso em 15 nov. 2013.

SALMON, Paul. Human Factors Design and Evaluation Methods Review. Southampton: HFi - DTC, 2004.

SAURO, Clare. Digitized Historic Costume Collections: Inspiring the Future While Preserving the Past. Journal of the American Society for Information Science and Technology, set.2009.

STEWART, Tekara S. Best practices of textile and clothing museum website development. Tese. lowa State University: Ames, 2009.

TULLIS, Tom; ALBERT, Bill. Measuring the user experience: Collecting, Analyzing, and Presenting Usability Metrics. Burlington: Morgan Kaufman, 2008. 